\title{
NOETHERIAN SUBSETS OF PRIME SPECTRA
}

\author{
CHARLES C. HANNA AND JON L. JOHNSON
}

\begin{abstract}
If $X$ is a noetherian subspace of Spec $R$, the set of primes of $R[x]$ lying over $X$ is also noetherian. A simple consequence is the theorem of Ohm and Pendleton that a ring module-finite over a $j$-noetherian ring is $j$-noetherian.
\end{abstract}

Let $R$ be a commutative ring with identity, $X$ an arbitrary subspace of Spec $R$, and $T$ a commutative $R$-algebra. Our main result is ultimately a version of the Ritt basis theorem [4, p. 14] and [3, Theorem 7] as generalized by Kaplansky [1, Theorem 7.1]. It was developed as a generalization of Theorem 2.5 of $\mathrm{Ohm}$ and Pendleton [2] in which they show that $\operatorname{Spec} T$ is noetherian if $\operatorname{Spec} R$ is noetherian and $T$ is finitely generated as an $R$-algebra. Our proof is adapted from theirs. We also show that Theorem 3.6 of [2] is a simple consequence of our theorem.

Let $R$ be a (commutative) ring, $X$ a subspace of $\operatorname{Spec} R$, and $I$ an ideal of $R$. The $X$-radical of $I$ is the intersection of the elements of $X$ which contain $I . I$ is $X$-finite if it has the same $X$-radical as some finitely-generated ideal $I_{0}$ contained in $I$. It is not difficult to check that $X$ is noetherian (a.c.c. on open subsets) if and only if every ideal is $X$-finite (see [2, Proposition 2.1]). Note that it is possible for every ideal of a ring to have the same $X$-radical as some finitely-generated ideal without $X$ being noetherian. See [2, Example 2.10].

THEOREM. Let $R$ be a ring and $x$ an indeterminate. Let $X$ be a noetherian subspace of Spec $R$. Let $Y$ be the set of all prime ideals of $R[x]$ whose contractions to $R$ are in $X$. Then $Y$ is noetherian.

Proof. Suppose that $Y$ is not noetherian. Let $P$ be maximal among the ideals of $R[x]$ which are not $Y$-finite. It is routine to show that $P$ must be prime (see [2, Proposition 2.3]). We will show that $P$ must in fact be $Y$-finite; this contradiction will prove the Theorem.

Write $P_{0}$ for $P \cap R$. Since $P_{0}$ is $X$-finite, $P_{0} R[x]$ is $Y$-finite. Let $T$ be the ring $R[x] / P_{0} R[x]$. Let $\bar{P}$ be the image of $P$ in $T$, and let $\bar{Y}$ be the image of $Y$ in Spec $T$. It is easy to verify that $P$ is $Y$-finite if and only if $\bar{P}$ is $\bar{Y}$-finite.

We may assume then that $P_{0}$ is the zero ideal so that $R$ is an integral domain. Let $K$ be its quotient field. Then $P K[x]$ is a principal ideal; we may assume that it is generated by a nonzero element $f$ of $P$. If $s$ is the leading coefficient of $f$, division

Received by the editors July 16, 1982.

1980 Mathematics Subject Classification. Primary 13A17; Secondary 13B99.

Key words and phrases. Prime spectrum, Noetherian spectrum. 
shows that $P R_{s}[x]$ is already principal (and generated by $f$ ). Now $s$ is not in $P$, so $(s, P)$ is a $Y$-finite ideal. We can choose elements $a_{1}, \ldots, a_{n}$ in $R[x]$ and $b_{1}, \ldots, b_{n}$ in $P$ so that $I_{0}=\left(a_{1} s+b_{1}, \ldots, a_{n} s+b_{n}\right)$ has the same $Y$-radical as $(s, P)$. Let $I$ be the ideal generated by $f$ and the $b_{i}$ 's. $I$ is contained in $P$; we claim $I$ has the same $Y$-radical as $P$.

Suppose that $Q$ is an element of $Y$ which contains $I$. If $s$ is in $Q$ then $I_{0}$ is contained in $Q$, so that $(s, P)$ is contained in $Q$. If $s$ is not in $Q$ then $Q R_{s}[x]$ is a prime ideal of $R_{s}[x]$ which contains $f$ and therefore contains $P R_{s}[x]$. In either case $Q$ contains $P$ and so $P$ is $Y$-finite.

COROllary. Let $\varepsilon: R \rightarrow T$ be a homomorphism of rings which makes $T$ a finitelygenerated $R$-algebra. Let $X$ be a noetherian subspace of $\operatorname{Spec} R$, and let $Y$ be $\left\{Q \in \operatorname{Spec} T \mid \varepsilon^{-1}(Q) \in X\right\}$. Then $Y$ is noetherian.

Proof. By induction; subspaces of noetherian spaces are noetherian.

COROLlaRY. Let $R$ and $R^{\prime}$ be rings such that $R^{\prime}$ is a finitely-generated $R$-module. If $R$ is j-noetherian then so is $R^{\prime}$.

Proof. Any maximal ideal of $R^{\prime}$ contracts to a maximal ideal of $R$.

\section{REFERENCES}

1. I. Kaplansky, An introduction to differential algebra, Publ. Inst. Math. Univ. Nancago, Paris, 1957.

2. J. Ohm and R. L. Pendleton, Rings with noetherian spectrum, Duke Math. J. 35 (1968), 631-640.

3. W. H. Raudenbush, Jr., Ideal theory and algebraic differential equations, Trans. Amer. Math. Soc. 36 (1934), 361-368.

4. J. F. Ritt, Differential equations from the algebraic viewpoint, Amer. Math. Soc. Colloq. Publ., vol. 14, Amer. Math. Soc., Providence, R.I., 1932.

Department of Mathematics, United States Naval ACademy, Annapolis, Maryland 21402

Department of Mathematics, Elmhurst College, Elmhurst, Illinois 60126 